ARTICLE

Received 17 Dec 2015 | Accepted 23 Mar 2016 | Published 26 Apr 2016

DOI: $10.1038 /$ ncomms11429

OPEN

\title{
Protein-directed self-assembly of a fullerene crystal
}

\author{
Kook-Han Kim', Dong-Kyun Ko르. Yong-Tae Kim¹, Nam Hyeong Kim¹, Jaydeep Paul ${ }^{3}$, Shao-Qing Zhang ${ }^{4,5}$, \\ Christopher B. Murray ${ }^{6}$, Rudresh Acharya ${ }^{3}$, William F. DeGrado ${ }^{4}$, Yong Ho Kim ${ }^{1,7} \&$ Gevorg Grigoryan ${ }^{8}$
}

Learning to engineer self-assembly would enable the precise organization of molecules by design to create matter with tailored properties. Here we demonstrate that proteins can direct the self-assembly of buckminsterfullerene $\left(\mathrm{C}_{60}\right)$ into ordered superstructures. A previously engineered tetrameric helical bundle binds $C_{60}$ in solution, rendering it water soluble. Two tetramers associate with one $\mathrm{C}_{60}$, promoting further organization revealed in a $1.67-\AA$ crystal structure. Fullerene groups occupy periodic lattice sites, sandwiched between two Tyr residues from adjacent tetramers. Strikingly, the assembly exhibits high charge conductance, whereas both the protein-alone crystal and amorphous $\mathrm{C}_{60}$ are electrically insulating. The affinity of $\mathrm{C}_{60}$ for its crystal-binding site is estimated to be in the nanomolar range, with lattices of known protein crystals geometrically compatible with incorporating the motif. Taken together, these findings suggest a new means of organizing fullerene molecules into a rich variety of lattices to generate new properties by design.

\footnotetext{
${ }^{1}$ SKKU Advanced Institute of Nanotechnology, Sungkyunkwan University, Suwon 16419, Korea. ${ }^{2}$ Department of Electrical and Computer Engineering, New Jersey Institute of Technology, Newark, New Jersey 07102, USA. ${ }^{3}$ School of Biological Sciences, National Institute of Science Education and Research, Bhubaneswar, Odisha 752050, India. ${ }^{4}$ Department of Pharmaceutical Chemistry and Cardiovascular Research Institute, University of California, San Francisco, California 94158, USA. ${ }^{5}$ Department of Chemistry, University of Pennsylvania, Philadelphia, Pennsylvania 19104, USA. ${ }^{6}$ Department of Chemistry and Materials Science and Engineering, University of Pennsylvania, Philadelphia, Pennsylvania 19104, USA. ${ }^{7}$ Center for Neuroscience Imaging Research, Institute for Basic Science (IBS), Suwon 16419, Korea. ${ }^{8}$ Department of Computer Science and Department of Biological Sciences, Dartmouth College, Hanover, New Hampshire 03755, USA. Correspondence and requests for materials should be addressed to R.A. (email: rudresh.acharya@niser.ac.in) or to W.F.D. (email: william.degrado@ucsf.edu) or to Y.H.K. (email: yhkim94@skku.edu) or to G.G. (email: gevorg.grigoryan@dartmouth.edu).
} 
P rogrammable self-assembly of molecular building blocks is a highly desirable way of achieving bottom-up control over novel functions and materials. Applications of molecular assemblies are well explored in the literature, ranging from optoelectronic $^{1,2}$, magnetic ${ }^{3}$, and photovoltaic ${ }^{4}$ devices to chemical and bioanalytical sensing ${ }^{5}$, and medicine ${ }^{6}$. However, it has been a daunting challenge to quantitatively describe and control the driving forces that govern self-assembly, particularly given the broad range of molecular building blocks one would like to organize. In this respect, nature's self-assembling macromolecules hold considerable promise as standard chassis for encoding precise organization. By learning to engineer the assembly of these molecules, myriad other molecular building blocks can be coorganized in desired ways through non-covalent or covalent attachment. The protein polymer is a particularly attractive candidate for a standard assembly chassis given its rich chemical alphabet, diversity of available assembly geometries, broad ability to engage other molecular moieties, and the possibility of engineered function. Considerable progress has been made in the area of engineering protein assemblies ${ }^{7,8}$, using either computational $^{9-14}$ or rational approaches ${ }^{15-18}$, but the problem remains a grand challenge. A major difficulty lies in accounting for the enormous continuum of possible assembly geometries available to proteins to engineer a sequence that predictably prefers just one. General design principles, which provide predictive rules of assembly, are thus of enormous utility in limiting the geometric search space and enabling robust design ${ }^{11,19}$.

In this work, we demonstrate the first ever high-resolution structure of co-assembly between a protein and buckminsterfullerene $\left(\mathrm{C}_{60}\right)$, which suggests a simple structural mode for proteinfullerene co-organization. Three separate crystal structures, resolved to $1.67,1.76$ and $2.35 \AA$, reveal a protein lattice with $\mathrm{C}_{60}$ groups occupying periodic sites wedged between two helical segments, each donating a Tyr residue. A half site of the motif is estimated to have nM-scale affinity for $\mathrm{C}_{60}$, such that binding of fullerene appears to direct the organization of protein units in the co-crystal. The assembly exhibits a nm-spaced helical arrangement of fullerenes along a crystallographic axis, endowing the crystal with electrical conductance properties. We closely investigate the interfacial geometry of the $\mathrm{C}_{60}$-binding motif, finding it to be common among protein crystal lattices. $\mathrm{C}_{60}$ and its derivatives have been previously reported to interact with several proteins ${ }^{20-25}$, although a high-resolution structure of a protein- $\mathrm{C}_{60}$ has been lacking. Still, prior evidence of interaction indicates that fullerenes and proteins are compatible as materials. This, together with the simple (and naturally recurrent) geometry of the $\mathrm{C}_{60}$-binding motif we discover, suggests that it may be possible to use the structural principles emergent from our study to generate a variety of $\mathrm{C}_{60}$-protein co-assemblies to further explore and exploit the properties of fullerenes ${ }^{26}$.

\section{Results}

C60-binding peptide organizes fullerenes. As a candidate for organizing $\mathrm{C}_{60}$, we considered a peptide we had designed in a previous study (sequence in Fig. 1a), which forms an antiparallel coiled-coil tetramer at $\mu \mathrm{M}$ concentrations (Protein Data Bank, PDB, entry 3S0R $)^{11}$. Two key properties appear to make the peptide suitable for assembling $\mathrm{C}_{60}$. First, the single aromatic residue in its sequence, tyrosine at position 9 , is exposed and available for potential $\mathrm{C}_{60}$ binding ${ }^{23,27}$. Second, the peptide appears to have an exceptionally low barrier to crystallization, forming X-ray diffraction quality crystals within hours. Inter-tetramer contacts within the resulting lattice are not extensive (Supplementary Fig. 1), suggesting that the ease of crystallization may be due to an innately low penalty for freezing out conformational degrees of freedom.

Upon sonication, $\mathrm{C}_{60}$ was readily solubilized in an aqueous solution of the peptide, hereafter referred to as COP ( $\mathrm{C}_{60}$-organizing peptide), but not buffer-only solutions. The resulting $\mathrm{C}_{60}-\mathrm{COP}$ suspensions, stable after centrifugation for at least 3 months (not monitored thereafter), produced characteristic absorbance spectra revealing the presence of both protein and the fullerene (Fig. 1b). Size-exclusion chromatography (SEC) of COP alone was consistent with its tetrameric oligomerization state (black in Fig. 1c). On the other hand, upon the solubilization of $\mathrm{C}_{60}$, an additional peak appeared in the chromatogram, corresponding to a species of molecular mass approximately that of a dimer of tetramers (red in Fig. 1c; Supplementary Fig. 2). This suggests that the solubilization of $\mathrm{C}_{60}$ occurs in a structurally specific manner with a change in oligomerization state of COP.

Despite COP's propensity to crystallize, attempts to co-crystallize $\mathrm{C}_{60}$ with COP were not met with success. The $\mathrm{C}_{60}-\mathrm{COP}$ suspensions did form crystals, but these appeared to be devoid of fullerene. We reasoned that this could be due to an insufficient amount of solubilized $\mathrm{C}_{60}$, such that not all binding sites on COP would be saturated and the protein-only species would selectively crystallize. Indeed, a rough estimate based on the $\mathrm{C}_{60}$-COP ultraviolet spectrum (Fig. 1b) and molar absorptivity of $\mathrm{C}_{60}$ at $340 \mathrm{~nm}$ taken from water/poly(vinylpyrrolidone) suspensions $^{28}$, suggests one molecule of $\mathrm{C}_{60}$ for $\sim 24$ COP tetramers (see Methods). To address this issue, we produced solutions of COP with $\mathrm{C}_{60}$ pyrrolidine Tris-acid $\left(\mathrm{C}_{60} \mathrm{Sol}\right.$; Supplementary Fig. 3), a more water-soluble analogue of $\mathrm{C}_{60}$ (solubility $0.002-0.005 \mathrm{mg} \mathrm{ml}^{-1}$ at $\mathrm{pH} 7.4$ ). The SEC chromatogram of the $\mathrm{COP}-\mathrm{C}_{60}$ Sol suspension again clearly shows two peaks-one corresponding to COP alone and another with apparent molecular weight corresponding to a dimer of COP tetramers (compare black and blue traces in Fig. 1c; also Supplementary Fig. 2). Further, absorbance at $340 \mathrm{~nm}$ (specific to the fullerene) clearly demonstrates that all of $\mathrm{C}_{60} \mathrm{Sol}$ elutes in the second (octameric) peak, arguing for a specific structure-based association (top plot, blue trace Fig. 1c). These results are further supported by analytical ultracentrifugation (AUC) sedimentation equilibrium experiments at a range of concentrations, showing tetramer-octamer equilibrium for $\mathrm{C}_{60} \mathrm{Sol}-\mathrm{COP}$ solutions (with a dissociation constant of $118 \mu \mathrm{M})$, whereas a single-species monomer model is sufficient for COP alone (Supplementary Methods).

Crystals from the resulting suspension grew within $24 \mathrm{~h}$ in several conditions, and three separate structures of the $\mathrm{C}_{60}$ Sol-COP complex were resolved to $1.67,1.76$ and $2.35 \AA$, respectively (Fig. 1d-g; Supplementary Figs 5-6; Table 1). To our knowledge, these represent first high-resolution structures of a protein-fullerene complex. As in the protein-only structure, COP forms a canonical tetrameric anti-parallel coiled coil ${ }^{29}$. Each tetramer presents four tyrosine residues (one per monomer) in exterior c positions of the coiled-coil heptad, and each of these engages a $\mathrm{C}_{60}$ moiety. One $\mathrm{C}_{60}$ is wedged between two Tyr residues donated by adjacent tetramers, such that two tetramers are needed to coordinate one $\mathrm{C}_{60}$ (Fig. 1f). This arrangement fits well with the apparent octameric peak in the SEC chromatogram and AUC profiles of $\mathrm{C}_{60}$ Sol-COP (Fig. 1c; Supplementary Fig. 4), suggesting that higher-order organization begins already in solution before crystallization. The water-solubilizing Tris-acid side chain of $\mathrm{C}_{60} \mathrm{Sol}$ is not visible in the electron density map. The group likely points into the solvated inner channel of the crystal and is highly mobile as the $\mathrm{C}_{60}$ core rapidly rotates around its centre. This is consistent with the intended role of the side chain, to increasing the solubility of the fullerene, whereas the $\mathrm{C}_{60}$ core appears responsible for the specific packing arrangement. 

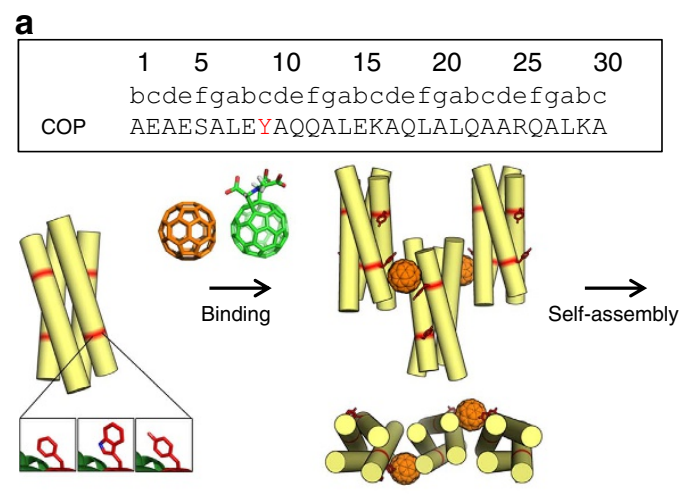

b

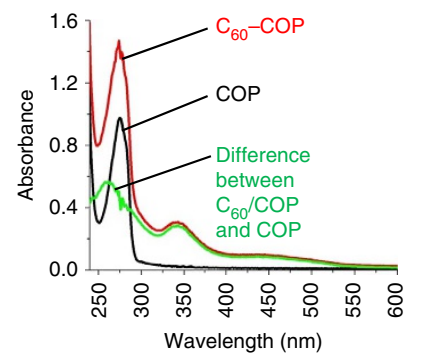

c

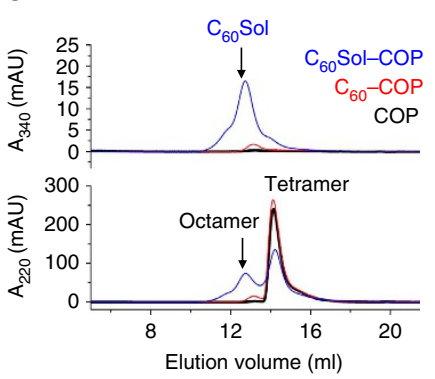

d

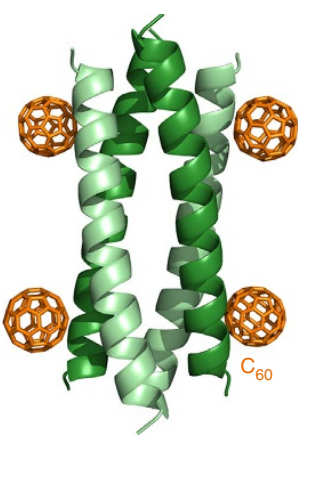

e

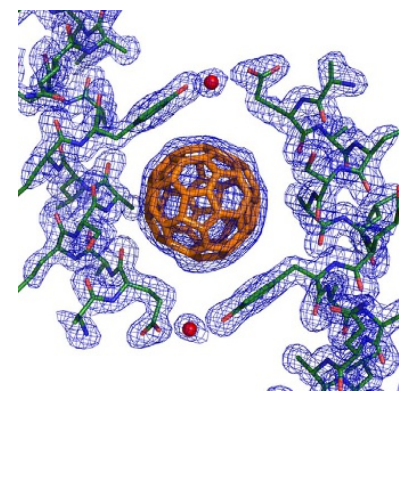

$f$

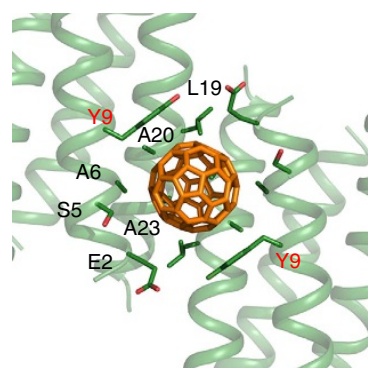

g

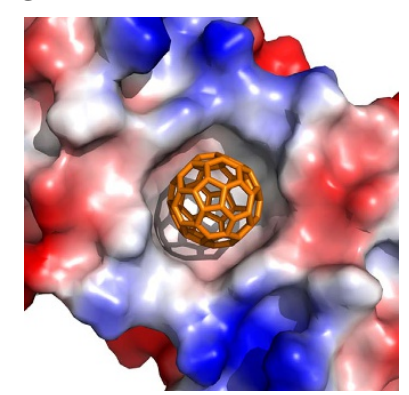

Figure 1 | Protein/ $\mathbf{C}_{60}$ super-assembly. (a) COP, a stable tetramer in isolation, interacts with $\mathrm{C}_{60}$ moieties by means of a surface-binding site that includes Tyr residues (other aromatic side chains also likely admissible), and further self-assembles into a co-crystalline array with fullerene. (b) Ultraviolet absorption spectra of a $\mathrm{C}_{60} / \mathrm{COP}$ suspension and $\mathrm{COP}$ alone demonstrate that primitive fullerene (green) dissolves in the aqueous phase in the presence of protein. (c) SEC traces of COP alone or in association with $\mathrm{C}_{60}$ or $\mathrm{C}_{60}$ Sol. Top and bottom plots show absorbances at 340 and $220 \mathrm{~nm}$, respectively. The lowerretention peaks arising due the addition of $\mathrm{C}_{60}$ or $\mathrm{C}_{60} \mathrm{Sol}$ are consistent with the molecular weight of a COP octamer (for example, dimer of tetramers; Supplementary Fig. 10). (d) Each COP tetramer in the $\mathrm{C}_{60}$ Sol-COP crystal is associated with four fullerenes (one per chain), each fullerene being wedged between two adjacent COP tetramers, for an overall stoichiometry of two fullerenes for one COP tetramers. (e) Omit map ( $2 F_{\mathrm{o}}-F_{\mathrm{c}}$, contoured at $\left.1.2 \sigma\right)$ showing electron density of the $\mathrm{C}_{60}$ group (orange sticks) sandwiched via $\pi-\pi$ stacking between Tyr residues from adjacent COPs. (f) Residues involved in $\mathrm{C}_{60}$ coordination are shown with sticks and labelled. (g) Surface representation of the $\mathrm{C}_{60}$ coordination site, coloured by relative in vacuo electrostatic potential (red to blue corresponds to negative-to-positive relative potentials).

The interaction between COP and the fullerene group involves non-polar contacts (Supplementary Fig. 7a), with $\mathrm{C}_{60}$ fitting perfectly into a symmetric hydrophobic cavity created by helices of two adjacent COP tetramers. The dominant contact appears to be the $\pi-\pi$ aromatic stacking between $\mathrm{C}_{60}$ and $\mathrm{Tyr} 9$, donated by each tetramer, while several aliphatic carbons line the pocket to further support the bound pose (Supplementary Fig. 7a; Fig. 1f,g). Interestingly, the conformation of COP itself is largely unchanged between the protein-only and protein/fullerene structures (Fig. 2a). Indeed, the $\mathrm{C} \alpha$ root mean squared deviation (r.m.s.d.) between the two tetramers is only $0.68 \AA$ (over 120 atoms; Fig. 2a,b), the full-backbone r.m.s.d. is $0.75 \AA$ (over 600 atoms), and the two structures represent coiled coils with essentially identical Crick parameters ${ }^{30}$ (see Methods; Supplementary Table 1). On the other hand, there is considerable difference in the arrangement of COP tetramers in the corresponding crystals: $\mathrm{COP}$ alone assembles into a body-centered cube, while $\mathrm{C}_{60} \mathrm{Sol}-$ $\mathrm{COP}$ has a honeycomb structure with parallel hexameric channels (Fig. 2c,d). This, together with the fact that neither of the crystals exhibit what would appear to be strong protein-protein interactions between tetramers, argues that the $\mathrm{C}_{60}$ group is chiefly responsible for driving the assembly of COP tetramers into the pattern observed in the co-crystal. In fact, in the fullerene bound structure, no contacts between adjacent COP tetramers occur outside of the $\mathrm{C}_{60}$-binding site. Furthermore, the three different $\mathrm{COP}-\mathrm{C}_{60} \mathrm{Sol}$ co-crystals obtained under different conditions (see Methods) all produced effectively identical binding interfaces and assembly geometries, arguing that fullerene may have a strong preference for the observed coordination geometry.

Helix-helix motif expected to bind fullerene tightly. We next ask whether the helix(Tyr)- $\mathrm{C}_{60}$-helix(Tyr)-binding mode could serve as a general co-organizer of proteins and fullerene. A necessary (but not sufficient) condition for this is that the motif would need to provide sufficient binding energy to drive assembly into a variety of desired arrangement. So we sought to quantify the affinity of $\mathrm{C}_{60}$ for the identified binding site. Direct equilibrium measurement of $\mathrm{C}_{60}$-protein association is complicated by the exceedingly low solubility of $\mathrm{C}_{60}$ in aqueous solution. Even the $\mathrm{C}_{60}$ Sol derivative has limited water solubility, hampering binding studies. We thus turned to explicit-solvent molecular dynamics simulations to characterize the strength of $\mathrm{COP}-\mathrm{C}_{60}$ association. The observed binding mode is a ternary interaction between two COP tetramers and one fullerene. To simplify the analysis, we concentrated on one half site of the symmetric binding pocket, looking to characterize the affinity of one $\mathrm{C}_{60}$ for one COP. Using the crystal structure as the starting bound configuration, thermodynamics of binding was characterized using a modification of the double-decoupling method $^{31}$ in conjunction with the free energy perturbation (FEP) approach 32 (see Methods; Supplementary Fig. 8). A total simulation time of $336 \mathrm{~ns}$ permitted accurate monitoring of 
Table 1 | Statistics on data collection and refinement of $\mathrm{C}_{60}$ Sol-COP complex.

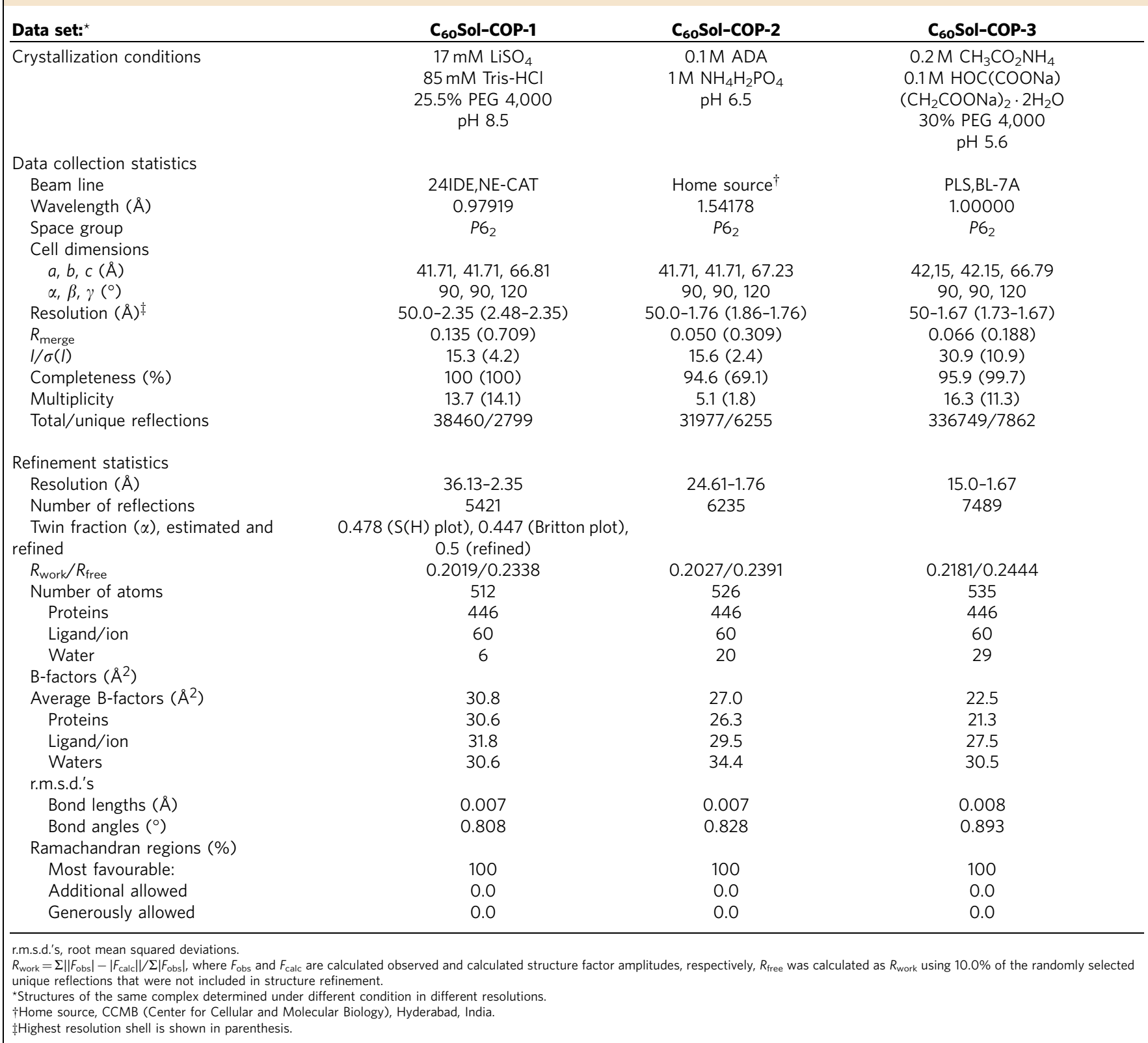

convergence, with the standard-state free energy of $\mathrm{C}_{60}-\mathrm{COP}$ binding estimated at $-9.8 \pm 0.3 \mathrm{kcal} \mathrm{mol}^{-1}$. This corresponds to a dissociation constant in the range of $40-100 \mathrm{nM}$, confirming the suspicion that $\mathrm{C}_{60}$ binding provides substantial energy to drive the assembly of COP units. In fact, the true energetic contribution of $\mathrm{C}_{60}$ is likely even larger as some positive cooperativity between the two motif half sites would be expected due to direct (albeit not extensive) favourable protein-protein interactions. Interestingly, we find that the $\pi-\pi$ stacking between $\mathrm{C}_{60}$ and Tyr9 is not sufficient to explain the strong interaction, as the affinities of $\mathrm{C}_{60}$ for an isolated Tyr residue (acetylated and methylamidated at the $\mathrm{N}$ - and $\mathrm{C}$ termini, respectively) or a $\mathrm{Tyr}$ side-chain analogue ( $p$-methylphenol) are estimated to be in the $\mathrm{mM}$ range (Supplementary Fig. 8b,c). Thus, additional aliphatic contacts in the binding pocket are essential for the collective binding mode and the high affinity.
Fullerene-binding motif composed of designable elements. Another necessary property of a generic protein-fullerene organizing motif is that it must be 'designable' in the context of a multitude of protein lattices-that is, the required geometry should be easily achievable with natural amino-acid sequences. Using the structural search engine MASTER ${ }^{33}$, we found that all of the interfaces involved in the motif are indeed highly abundant in nature (and are thus necessarily designable), with emergent sequence preferences in agreement with the corresponding region of COP (Methods; Supplementary Fig. 7b). Further, even the entire binding motif, composed of four disjoint helical segments that account for all contacts with $\mathrm{C}_{60}$ in the supercrystal, has precedence in PDB lattices. In fact, within a homology/redundancy-pruned subset of the $\operatorname{PDB}(13,400$ entries), we found 180 unique instances of matching geometries (below 1.9 ̊̊ backbone r.m.s.d., computed over 112 atoms) within 21 unique lattices (Supplementary Fig. $7 \mathrm{c}$ ). That is, $\sim 0.15 \%$ of 
a

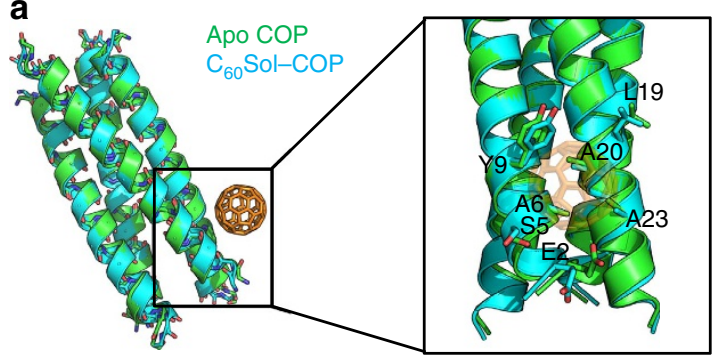

b

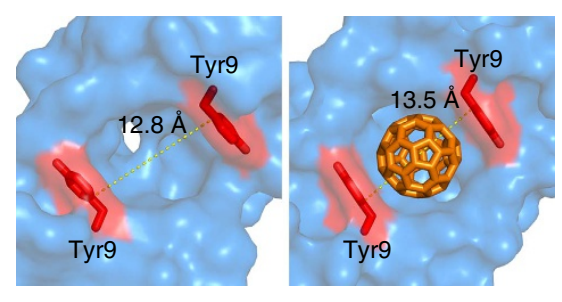

C
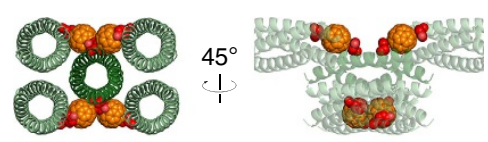

$90^{\circ}-\mathrm{-}$

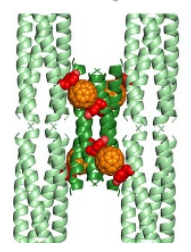

$90^{\circ}$

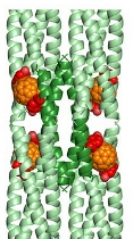

d

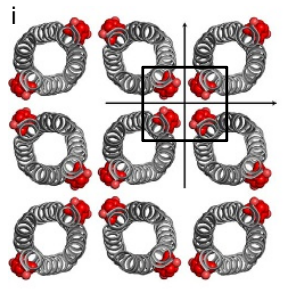

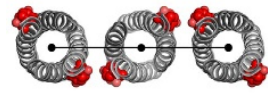

Apo COP

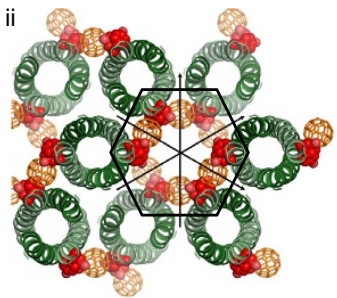

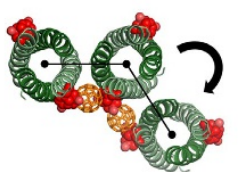

$\mathrm{C}_{60}$ Sol-COP

Figure 2 | COP crystal adjusts to incorporate fullerene. (a) Superposition of apo COP (green) and $\mathrm{C}_{60}$ Sol-COP (cyan) shows no significant structural changes in the helix bundle. Side-chain differences around the fullerene-binding site are highlighted in the box. (b) The distance between aromatic Tyr9 residues adjusts in $\mathrm{C}_{60} \mathrm{Sol}-\mathrm{COP}$ to incorporate the fullerene. (c) Different views of the COP-fullerene lattice. (d) Significant changes in the crystal lattice between $\mathrm{COP}$ alone and $\mathrm{C}_{60}$ Sol-COP structures. Viewed from the top, $\mathrm{C}_{60}$ Sol-COP forms a honeycomb structure (ii), whereas apo COP exhibits a tetrameric cube pattern (i).

proteins in the PDB already exhibit backbone geometries similar to the one housing a bound $\mathrm{C}_{60}$ in the co-crystal, suggesting that it may be possible to engineer a variety of fullerene/protein co-assemblies by perturbing sequences of existing parent proteins. Supplementary Fig. 7c shows examples of such putative co-assemblies, where $\mathrm{C}_{60}$ is computationally placed into existing lattices matching the binding motif, giving diverse $\mathrm{C}_{60}$-to- $\mathrm{C}_{60}$ distances and lattice arrangements. Of course, the design of such assemblies will involve not only the placement of a $\mathrm{C}_{60}$-binding motif, but also any appropriate accommodating changes to surrounding amino acids. Further, there is no guarantee that the crystal form will not change upon these perturbations. Still, that our identified motif appears 'canonical' in terms of its constituent protein-protein interfaces is encouraging for future design applications.

Fullerene-protein crystal has emergent electronic properties. The honeycomb structure of $\mathrm{C}_{60}$ Sol-COP is intriguing from the perspective of its electronic properties. Within the helical arrangement of fullerenes, inter- $\mathrm{C}_{60}$ distances appear sufficiently close for potential long-range electronic transfer, especially given the organized nature of the surroundings ${ }^{34,35}$ (Fig. $3 a-c$ ). For this reason, we sought to characterize the electrical conductance of the co-crystal. Current-voltage $(I-V)$ characteristic of disordered $\mathrm{C}_{60}$ films showed high electrical resistance of $2.24 \times 10^{11} \Omega$ (Fig. 3d and Supplementary Fig. 9). In addition, COP-alone crystals or crystal buffer similarly showed high resistance, with only $5-10 \mathrm{pA}$ of currents measured with up to $20 \mathrm{~V}$ of voltage sweep. On the other hand, $\mathrm{C}_{60}$ Sol-COP supercrystals (of similar dimension as protein-alone crystals) exhibited high electrical conductance $\left(1.40 \times 10^{-7} \mathrm{~S}\right.$, corresponding to resistance of $7.14 \times 10^{6} \Omega$ ) with at least four orders of magnitude higher currents than in any of the controls (Fig. 3d). We speculate that the periodic arrangement of fullerene groups in the co-crystal may facilitate electron wave delocalization over the assembly. This would promote coherent electron transport through the structure with the carrier mobility expected to be several orders of magnitude higher than in disordered systems characterized by hopping transport ${ }^{36}$. Inter-fullerene nearest-neighbour distances in the $\mathrm{C}_{60}$ Sol-COP supercrystal alternate between 1.2 and $1.7 \mathrm{~nm}$ (Fig. 3c). For comparison, strong electron wave delocalization was previously observed when the nearest-neighbour distance approached $\sim 1.5 \mathrm{~nm}$ in one-dimensional fullerene wires ${ }^{37}$. An alternative explanation of the observed conductive property is that the hexameric channels in the co-crystal may contain unattached/disordered fullerene moieties that are free to diffuse in the channel and can shuttle electrons between ordered in-lattice fullerenes. In either case, as shown in Fig. 3d (yellow dots), destruction of crystalline order (by placement in vacuum) results in very high electrical resistance. In fact, the current measured here is even lower than that of the disordered $\mathrm{C}_{60}$ film. This indicates that the high conductivity of the $\mathrm{C}_{60}$ Sol-COP supercrystal is not a trivial property of crystal dimension and/or molecular composition, but rather originates from specific electronic coupling/delocalization in the assembly.

\section{Discussion}

The aim of programmable self-assembly is to anticipate and harness unique collective properties that arise from precisely organized molecular building blocks. To this end, achieving atomic-level precision is crucial. This work demonstrates the first atomic resolution structures of a fullerene-protein assembly, establishing the feasibility of creating such objects, and further suggests a possible design principle for engineering such 


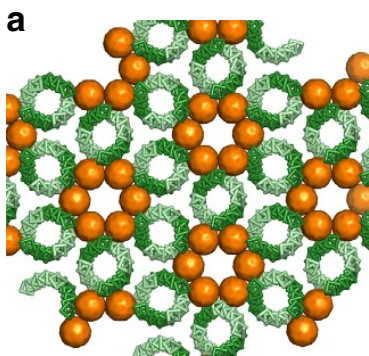

b

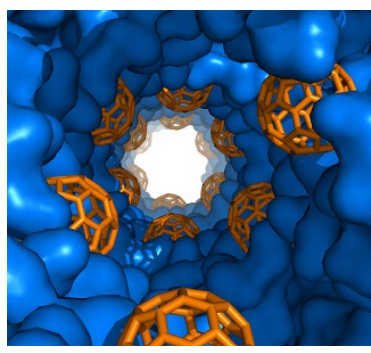

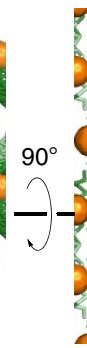

C

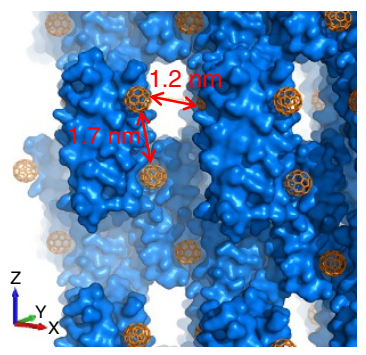

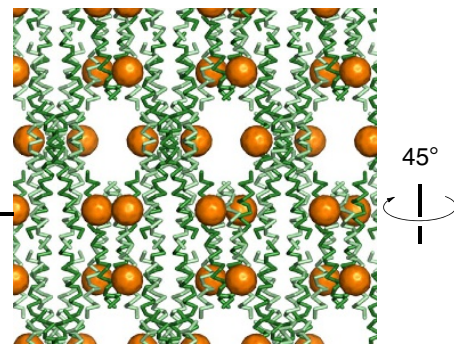

d

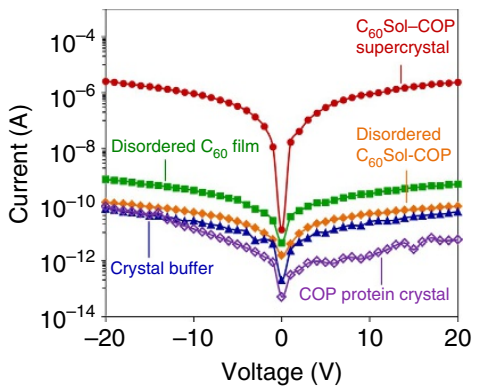

Figure 3 | Assembly of fullerenes endows crystal with electronic transport capabilities. (a) Three views of the $\mathrm{C}_{60} \mathrm{Sol}_{-\mathrm{COP}}$ crystal lattice. (b) $\mathrm{C}_{60}$ groups are arranged in a helical manner along parallel inner channels in the assembly. (c) A side view of the channel showing nearest-neighbor inter- $\mathrm{C}_{60}$ distances. (d) Semi-logarithmic current-voltage characteristic of $\mathrm{C}_{60}$ Sol-COP supercrystal (red dots) and disordered $\mathrm{C}_{60}$ Sol-COP (orange diamonds). Disordered $\mathrm{C}_{60}$ film dried from a bare $\mathrm{C}_{60}$ /toluene solution (green squares), crystal buffer solution (blue triangles) and a COP-alone protein crystal (violet open circles) were also characterized as controls.

assemblies in general. How robust the discovered $\mathrm{C}_{60}$-binding motif is towards designing novel assemblies will need to be tested through a number of future design studies. However, the straightforward manner in which self-organization arose in our case, the simplicity of the $\mathrm{C}_{60}$-organizing motif in the lattice, together with its high affinity and the ubiquity of associated interfaces in natural protein lattices, are certainly promising with respect to the general applicability of the design principle. Our work also demonstrates the potential utility of exploring $\mathrm{C}_{60}$ /protein co-organization, as derived supercrystals already showed synergistic charge conductance properties. Taken together, these results point to an exciting direction of inquiry towards generating protein-fullerene assemblies for the study and design of novel properties.

\footnotetext{
Methods

Peptide synthesis and purification. Peptides were synthesized by CEM Discover microwave synthesizer using Fmoc chemistry at $100-\mu$ mol scales. The Fmoc protecting group was removed by piperidine/dimethylformamide solution $(20 / 80 \mathrm{v} / \mathrm{v})$; at each coupling step reactants were added with the amino acid:HBTU:DIEA:resin ratio of 5:4.9:10:1. Products were cleaved from the H-Rink Amide-ChemMatrix (PCAS, $0.53 \mathrm{mmolg}^{-1}$ loading) in a cleavage cocktail solution (trifluoroacetic acid (TFA)/triisopropyl silane/deionized water, 95/2.5/2.5 v/v) for $2 \mathrm{~h}$ and the remaining solution was vapourized with $\mathrm{N}_{2}$ gas. Peptide was precipitated with cold diethyl ether (Aldrich) and dried in vacuum. After dissolving the peptides in DI H2O, purification proceeded by preparative reverse-phase high-performance liquid chromatography (Waters prep 150 LC System) using preparative C4 column (XBridge BEH300 Prep $\mathrm{C} 45 \mathrm{um})$ and a linear gradient of buffer A $(99.9 \% \mathrm{H} 2 \mathrm{O}$ and $0.1 \%$ TFA) and buffer B ( $90 \%$ acetonitrile, $9.9 \% \mathrm{H} 2 \mathrm{O}$ and $0.1 \%$ TFA). Molecular mass of the peptide was confirmed by matrix-assisted laser desorption/ionization-time of flight mass spectrometry (Bruker Ultraflex III). Products had over $95 \%$ purity.
}

Preparation of peptide/fullerene solutions. Samples were prepared with $8 \mathrm{mg} \mathrm{ml}^{-1}$ protein solution (COP) in $25 \mathrm{mM}$ Tris $\mathrm{pH} 8.0$ buffer solution and $1 \mathrm{mg}$ $\mathrm{C}_{60}$ or $\mathrm{C}_{60}$ pyrrolidine Tris-acid (Aldrich). Fullerene powder was mixed with pre-made $0.2 \mathrm{ml}$ of $8 \mathrm{mg} \mathrm{ml}^{-1}$ protein solution in $25-\mathrm{mM}$ Tris $\mathrm{pH} 8.0$. The sample was then tip-sonicated (QSonica, Q125, 1/8th inch tip) on an ice bath for $5 \mathrm{~min}$ to be saturated of fullerene. Ice-bath cooling was to prevent excessive sample heating and destabilization of protein structure. The sonicated samples were warmed up to room temperature and centrifuged at $14,500 \mathrm{~g}$ for $10 \mathrm{~min}$ (Eppendorf, Centrifuge 5430R).
Ultraviolet-visible absorption spectroscopy. Ultraviolet absorption spectra of the COP alone and $\mathrm{C}_{60} / \mathrm{COP}$ were recorded using a Hewlett Packard 8453 spectrometer in $1 \mathrm{~cm}$ Hellma Quartz SUPRASIL (QS) cells. The COP and $\mathrm{C}_{60} / \mathrm{COP}$ were prepared in a buffer of $20 \mathrm{mM}$ sodium phosphate, $100 \mathrm{mM} \mathrm{NaCl}$ and $\mathrm{pH}$ 7.5. Ultraviolet-visible spectra of $\mathrm{C}_{60} / \mathrm{COP}$ and COP were used to roughly estimate the concentration of solubilized fullerene by absorbance at $340 \mathrm{~nm}$ (the molar absorptivity of $49,000 \mathrm{M}^{-1} \mathrm{~cm}^{-1}$ was used for $\mathrm{C}_{60}$, (ref. 28). The resulting molar concentration of solubilized $\mathrm{C}_{60}$ in the $\mathrm{C}_{60} / \mathrm{COP}$ solution was $6.22 \mu \mathrm{M}$ (compared with COP at $585 \mu \mathrm{M}$ in the same solution).

Size-exclusion chromatography. Size-exclusive gel filtration elution profiles were obtained using a Superdex 75 10/300 GL column with a GE Healthcare fast performance liquid chromatography (FPLC) system (Amersham Pharmacia Biosystems). Peptides (at $200 \mu \mathrm{M}$ ) were prepared in a buffer of $20 \mathrm{mM}$ sodium phosphate, $100 \mathrm{mM} \mathrm{NaCl}$ and $\mathrm{pH} 7.5$ at room temperature. $200 \mu \mathrm{l}$ of each sample were loaded and eluted with the same buffer. The column was equilibrated in $20 \mathrm{mM}$ sodium phosphate, $100 \mathrm{mM} \mathrm{NaCl}$ and $\mathrm{pH} 7.5$ with a mobile phase flow rate of $0.5 \mathrm{ml} \mathrm{min}^{-1}$, and absorbance at 220,280 and $340 \mathrm{~nm}$ was recorded. Calibration curves were obtained using the molecular-weight standard kit, MWGF70 6,500-66,000 (Supplementary Fig. 10).

Analytical ultracentrifugation. Oligomerization states of $\mathrm{COP}$ and $\mathrm{C}_{60} \mathrm{Sol}-\mathrm{COP}$ were determined by equilibrium sedimentation performed at $25^{\circ} \mathrm{C}$ using a Beckman XL-I analytical ultracentrifuge. Both solutions were prepared in a buffer of $25 \mathrm{mM}$ Tris $\mathrm{pH}$ 8.0. Equilibrium radial concentration gradients at four different rotor speeds $(25,30,35$ and $40 \mathrm{~K}$ r.p.m.) were acquired as absorbance scans at $340 \mathrm{~nm}$ for $\mathrm{C}_{60} \mathrm{Sol}$ with COP and $280 \mathrm{~nm}$ for COP peptide alone. Data were globally fit to single-species or two-species models of equilibrium sedimentation by a nonlinear least-squares method using IGOR Pro (Wavemetrics), and the best-fitting model was accepted ${ }^{38}$. Supplementary Figure 4 shows sedimentation equilibrium profiles of $\mathrm{C}_{60} \mathrm{Sol}-\mathrm{COP}$ along with corresponding species distribution plots consistent with a tetramer-octamer equilibrium, whereas COP alone appears as a tight tetramer. This is consistent with results from SEC, shown in Fig. 1c and Supplementary Fig. 2.

Crystallization, data collection and processing. The first X-ray diffraction quality crystal ( $\mathrm{C}_{60}$ Sol-COP-1) was obtained by the hanging-drop vapour diffusion technique at $291 \mathrm{~K}$, over a period of 15 days in a $2 \mu \mathrm{l}$ drop consisting of $1: 1 \mathrm{v} / \mathrm{v}$ mixture of $1 \mathrm{mgml}^{-1}$ protein solution in $20 \mathrm{mM}$ sodium phosphate $/ 100 \mathrm{mM} \mathrm{NaCl}$ $\mathrm{pH} 7.5$ buffer and a reservoir solution of $17 \mathrm{mM}$ lithium sulfate monohydrate, 85 mM Tris-hydrochloride sodium pH 8.5, 25.5\% polyethylene glycol (PEG) 4,000, $25 \% \mathrm{v} / \mathrm{v}$ glycerol (Hampton Research sparse matrix). The crystal was flash-frozen, and diffraction data were collected at the 24-ID-E NE-CAT beamline at the 
Argonne National Laboratory. Data sets were indexed and integrated with MOSFLM $^{39,40}$, and scaled using SCALA3 (Collaborative Computational Project, Number 4,1994$)^{41}$. Diffraction data were recorded to a maximum resolution of $2.35 \AA$ (Table 1).

Subsequent crystallization attempts were performed with higher concentrations of the $\mathrm{C}_{60} \mathrm{Sol} / \mathrm{COP}$ suspension, using commercially available sparse-matrix screens from Hampton Research and the hanging-drop vapour diffusion method at $295 \mathrm{~K}$. Diffraction-quality crystals of $\mathrm{C}_{60}$ Sol-COP $\left(\mathrm{C}_{60} \mathrm{Sol}-\mathrm{COP}-3\right)$ were obtained by mixing equal volumes of the $\mathrm{C}_{60} \mathrm{Sol}-\mathrm{COP}$ mixture at $8 \mathrm{mg} \mathrm{ml}^{-1}$ in $25 \mathrm{mM}$ Tris $\mathrm{pH} 8.0$ and reservoir solution consisting of $0.2 \mathrm{M}$ ammonium acetate, $0.1 \mathrm{M}$ sodium citrate tribasic dihydrate $\mathrm{pH} 5.6,30 \% \mathrm{w} / \mathrm{v}$ polyethylene glycol 4,000. Microcrystals grew within $24 \mathrm{~h}$, with larger oval-shaped crystals appearing in several days (Supplementary Fig. 5). Crystals were cryoprotected using reservoir solution supplemented with an additional $30 \%$ (v/v) glycerol and were flash-cooled in liquid nitrogen. Diffraction data, extending to $1.67 \AA$ resolution, were collected at $100 \mathrm{~K}$ on beamline 7A equipped with an ADSC Quantum 270 CCD detector at Pohang Accelerator Laboratory (PAL, Pohang, Korea). The $\mathrm{C}_{60}$ Sol-COP complex crystal belonged to space group $P 6_{2}$, with unit cell parameters $a=b=42.1, c=66.7 \AA$, $\alpha=\beta=90.0$ and $\gamma=120.0^{\circ}$. Data were processed and scaled using the programs DENZO and SCALEPACK from the HKL-2000 program suite ${ }^{42}$. The Matthews coefficient ${ }^{43}$ for $\mathrm{C}_{60}$ Sol-COP was $2.54 \AA^{3} \mathrm{Da}^{-1}$ and the estimated solvent content was $51.5 \%$; there were two COP molecules and one $\mathrm{C}_{60} \mathrm{Sol}$ in an asymmetric unit.

In addition to the above, diffraction-quality crystals were also obtained in three other conditions (1.5 M ammonium sulfate, $0.1 \mathrm{M}$ Tris $\mathrm{pH} 8.5,12 \% \mathrm{v} / \mathrm{v}$ glycerol; $0.1 \mathrm{M}$ HEPES-Na $\mathrm{pH} 7.5,0.8 \mathrm{M}$ potassium sodium tartrate tetrahydrate; and $0.1 \mathrm{M}$ $N$-(2-acetamido)iminodiacetic acid, $N$-(carbamoylmethyl)iminodiacetic acid (ADA) buffer pH 6.5, $1 \mathrm{M}$ ammonium phosphate dibasic), in each case yielding identical unit cell and space group, thus showing the same assembly geometry. Crystals grown under the latter condition diffracted to $1.76 \AA$ at a home source ( $\mathrm{C}_{60}$ Sol-COP-2).

Structure solution and refinement. For all the data sets, structure determination was carried out by molecular replacement using the programme PHASER ${ }^{44}$. The Matthews coefficient suggested a dimeric helix in the asymmetric unit. Molecular replacement calculations were performed using the dimeric unit of a polyalanine model obtained from coordinates of previously solved crystal structure $3 \mathrm{SOR}$ as the search probe. The solution model was subjected for rigid body refinement followed by iterative model building and restrained refinement protocols implemented in Auto Build module of PHENIX ${ }^{45}$. All side chains were traced in the electron density map. During the course of map tracing, electron density for fullerene was clearly visible and modelled for refinement.

During data analysis, it was found that the crystal $\left(\mathrm{C}_{60} \mathrm{Sol}-\mathrm{COP}-1\right)$ was merohedrally twinned. The H-test results, $|H|=0.024$ (0.50 for untwinned and 0.0 for $50 \%$ twinned) and $H^{2}=0.001$ ( 0.33 for untwinned and 0.0 for $50 \%$ twinned), were indicative of merohedral twinning with the twin law (h, -h-k, $-\mathrm{l}$ ), where $H=\left|I_{1}-I_{2}\right| /\left|I_{1}+I_{2}\right|, I_{1}$ and $I_{2}$ are twin-related acentric reflections. The cumulative distribution of $\mathrm{H}^{46,47}$ and Britton plots ${ }^{48,49}$ estimated twin domain fraction $(\alpha)$ to be 0.478 and 0.447 , respectively.

As the estimated twin fraction was close to 0.5 , the data were not detwinned for further refinement. Instead, the refinement was carried out by refining both parameters of the model and twin fraction. The PHENIX ${ }^{45}$ refinement protocol, which implements this option, was used.

The PHENIX refinement protocol was used. Upon converging, the refinement strategy produced model with good $R_{\text {work }} / R_{\text {free }}$ statistics in each case (Table 1 ). Model building was further carried out manually using COOT $^{50}$. Structure figures were created using the programme PyMOL (Schrödinger, LLC). Crystallographic data statistics are summarized in Table 1.

Coiled-coil parameter fitting. Parameter fits were performed using CCCP (http://grigoryanlab.org/cccp) via the 'global symmetric' fit option, where ideal symmetry (in this case D2) is assumed ${ }^{30}$. The apo and $\mathrm{C}_{60}$-bound structures fit within 0.6 and $0.4 \AA$, respectively, indicating that they both closely resembled an ideal coiled coil. Key parameters are listed in Supplementary Table 1. Detailed parameter definitions and information on the fitting procedure can be found in reference $\mathrm{e}^{30}$.

Binding free-energy calculation. The NAMD 2.10 software package, developed by the Theoretical and Computational Biophysics Group in the Beckman Institute for Advanced Science and Technology at the University of Illinois at Urbana-Champaign $^{51}$, in conjunction with CHARMM22 force field ${ }^{52}$ was used for this study. A new atom type was created for the $\mathrm{C}_{60}$ carbon (CA60), which was identical to the aromatic carbon atom type in CHARMM22 (type CA) in all aspects except for the equilibrium CA60-CA60 bond length, which was set to $1.4392 \AA$ to match the experimentally observed average bond length in $\mathrm{C}_{60}$ (ref. 53). All simulations were performed in explicit TIP3P water; a padding of $8 \AA$ was used for initial solvation, with sodium/chloride counterions added to achieve charge neutrality as necessary. Periodic boundary conditions were applied and all simulations were performed in the NTP ensemble at $298.15 \mathrm{~K}$ and $1 \mathrm{~atm}$. Explicit calculation of long-range interactions was cutoff at $10 \AA$, with a switching function starting at $6 \AA$. Particle Mesh Ewald method was used to correct for long-range electrostatics ${ }^{54}$ and an analytical correction was used to capture long-range van der Waals interactions ${ }^{55}$. Pande and co-workers have shown that with these corrections, the 6/10 ^ non-bond cutoff schedule performed as well as longer cutoffs in free energy of solvation calculations ${ }^{56}$.

To compute the free energy of $\mathrm{C}_{60}-\mathrm{COP}$ association, we followed the double-decoupling framework outlined by McCammon and co-workers ${ }^{31}$. In this approach, one seeks to compute the free energy of decoupling the ligand (here $\mathrm{C}_{60}$ ) from the rest of the system when it is either bound to the receptor (here COP) or solvated by itself. The standard-state free energy of binding is then related to the difference between the two decoupling free energies, appropriately corrected for the standard-state concentration ${ }^{31}$. We sought to use the method of FEP to compute individual decoupling free energies, but the direct application of the method to $\mathrm{C}_{60}$ exhibited very strong hysteresis between forward and reverse simulations (that is, decoupling $\mathrm{C}_{60}$ and coupling it back, respectively). Because $\mathrm{C}_{60}$ is hollow, with enough space inside for several water molecules, as the molecule is decoupled, water rushes in to occupy the available space. However, during the reverse simulation, as $\mathrm{C}_{60}$ is coupled back to the system, water molecules tend to remain trapped inside the fullerene, leading to a very different end state. Note that use of the soft-core van der Waals scaling ${ }^{55}$, implemented in NAMD, does not resolve this issue as there is little to encourage water molecules to escape the core of fullerene as it is coupled back. This very large hysteresis meant that we could not claim good convergence (and hence accuracy) of either forward or reverse simulations.

To resolve this issue, we introduced an intermediate step in the $\mathrm{C}_{60}$ decoupling/coupling transformation, designed to provide reversibility, slightly adjusting the double-decoupling framework. The idea was to introduce an artificial atom, with a size to roughly match the radius of $\mathrm{C}_{60}$, which could be used to 'make room' for $\mathrm{C}_{60}$ before the molecule is coupled to the system. A new atom type was created, called C60D (for $\mathrm{C}_{60}$ 'dummy'), with a van der Waals radius of $4.5 \AA$ and a Lennard-Jones well depth of $-1.0 \mathrm{kcal} \mathrm{mol}^{-1}$. Because C60D is a single atom, and not hollow-like $\mathrm{C}_{60}$, the soft-core van der Waals potential will indeed gradually repel water molecules as $\mathrm{C} 60 \mathrm{D}$ appears. Thus, the two decoupling transformations were altered as follows:

$$
\mathrm{COP} \cdot \mathrm{C} 60_{\text {wat }}+\mathrm{C} 60 D_{\text {gas }} \stackrel{\text { step } 1}{\rightleftharpoons} \mathrm{COP} \cdot \mathrm{C} 60 D_{\text {wat }}+\mathrm{C} 60_{\text {gas }} \stackrel{\text { step2 }}{\rightleftharpoons} \mathrm{COP}+\mathrm{C} 60_{\text {gas }}+\mathrm{C}_{6} 0 \mathrm{D}_{\text {gas }}
$$

$$
\mathrm{C} 60 \text { wat }+\mathrm{C} 60 \mathrm{D}_{\text {gas }} \stackrel{\text { step } 1}{\rightleftharpoons} \mathrm{C} 60 \text { gas }+\mathrm{C} 60 \mathrm{D}_{\text {wat }} \stackrel{\text { step2 }}{\rightleftharpoons} \mathrm{C} 60_{\text {gas }}+\mathrm{C} 60 \mathrm{D}_{\text {gas }}
$$

where subscripts wat and gas indicate that the corresponding molecule is either fully coupled to the system (that is, in water) or fully decoupled from it (that is, in the gas phase), respectively. The initial state of transformation 1 involves the complex between COP and $\mathrm{C}_{60}\left(\mathrm{COP} \cdot \mathrm{C} 0_{\text {wat }}\right)$ and a decoupled $\mathrm{C} 60 \mathrm{D}\left(\mathrm{C} 60 \mathrm{D}_{\text {gas }}\right)$ overlapping the fullerene. The first step of the transformation involves decoupling $\mathrm{C}_{60}$ from the system, while C60D is coupled, such that the intermediate step has $\mathrm{C}_{60}$ in the gas phase $\left(\mathrm{C} 60 \mathrm{D}_{\text {gas }}\right)$ while $\mathrm{C} 60 \mathrm{D}$ is fully interacting with the system, occupying the fullerene-binding site $\left(\mathrm{COP} \cdot \mathrm{C} 60 \mathrm{D}_{\text {wat }}\right)$. The second step of the transformation then decouples $\mathrm{C60D}$ as well, such that the end state involves COP in solvent alone with both $\mathrm{C}_{60}$ and $\mathrm{C} 60 \mathrm{D}$ in the gas phase. Because gaseous C60D is present in both end states, its contribution to the total free-energy difference cancels, such that the net transformation still represents just the decoupling of $\mathrm{C}_{60}$. On the other hand, the presence of $\mathrm{C} 60 \mathrm{D}$ and the intermediate state address the reversibility of the transformation. Because the first step in the reverse direction involves coupling of $\mathrm{C} 60 \mathrm{D}$, room is created in the solvent before $\mathrm{C}_{60}$ is reintroduced and $\mathrm{C} 60 \mathrm{D}$ is once again decoupled in the second step. To prevent C60D from diffusing away from the binding site at any point in the simulation, harmonic restraints were applied between $\mathrm{C} 60 \mathrm{D}$, and $\mathrm{C} \gamma, \mathrm{C} \varepsilon_{1}$ and $\mathrm{C} \varepsilon_{2}$ atoms of the binding site Tyr (residue 9), with equilibrium distances of $6.7,6.7$ and $7.0 \AA$, respectively (taken from the crystal structure by initially placing C60D in the geometric centre of the bound $\mathrm{C}_{60}$ ), and a force constant of $10 \mathrm{kcal} \mathrm{mol}^{-1} \AA^{-2}$. Note that these restraints do not contribute to the FEP calculation (since their energy is independent of the coupling parameter) and their presence fully cancels between end states of the transformation. Another restraint was needed to make sure $\mathrm{C}_{60}$ does not diffuse far from the binding site when decoupled, which would create convergence difficulties. A harmonic restraint was applied between the centre of mass of $\mathrm{C}_{60}$ and C60D, with an equilibrium distance of zero and a force constant that increased from 0 to $10 \mathrm{kcal} \mathrm{mol}^{-1} \AA^{-2}$ as $\mathrm{C}_{60}$ was decoupled from the system. Specifically, if $\lambda$ is the FEP coupling parameter for the current window (with 0 and 1 corresponding to $\mathrm{C}_{60}$ being fully coupled and decoupled, respectively), the force constant used was $10 \cdot \lambda^{2} \mathrm{kcal} \mathrm{mol}^{-1} \AA^{-2}$. The energy of this restraint was accounted for in FEP calculations, so that the final free-energy change for transformation 1 represented the difference between a state where $\mathrm{C}_{60}$ is fully coupled and bound to COP and one where $\mathrm{C}_{60}$ is decoupled from the system, but harmonically restrained to remain in the vicinity of the binding site. To remove the influence of this restraint and correct for the standard state, this 
free-energy change was corrected by $R T \ln \left[C^{o}(2 \pi R T / k)^{3 / 2}\right]$, where $C^{\circ}$ is the standard-state concentration and $k$ is the force constant of the $\mathrm{C}_{60}$ restraint in the decoupled state (that is, $\left.10 \mathrm{kcal} \mathrm{mol}^{-1} \AA^{-2}\right)^{31,57}$.

Transformation 2 is similar to transformation 1, but with no protein. In the first step, $\mathrm{C}_{60}$ is decoupled from solvent as $\mathrm{C} 60 \mathrm{D}$ is coupled, whereas the second step decouples C60D. As with transformation 1, the influence of C60D cancels between the two end states, with the total free-energy difference corresponding to that of decoupling $\mathrm{C}_{60}$ from solvent. However, the intermediate step again renders the path reversible. As with transformation 1, here it was important for $\mathrm{C}_{60}$ and $\mathrm{C} 60 \mathrm{D}$ to be approximately coincident throughout the simulation (so that, for example, in the first step of the reverse simulation coupling of C60D creates a cavity in the right location within the solvent for $\mathrm{C}_{60}$ to couple into later). For this reason, a harmonic restraint was introduced between the centroid of $\mathrm{C}_{60}$ and $\mathrm{C} 60 \mathrm{D}$, with equilibrium distance of zero and a force constant of $10 \mathrm{kcal} \mathrm{mol}^{-1} \AA^{-2}$. Note that the contribution of this constant restraint cancels between the two end states (so the total free-energy change of transformation 2 is still that of decoupling $\mathrm{C}_{60}$ alone), and its energy does not influence FEP calculations.

Since $\mathrm{C}_{60}$ remains decoupled (and restrained to C60D) throughout step 2 of both transformations, it does not contribute to the free-energy change associated with these steps. For this reason, $\mathrm{C}_{60}$ need not be explicitly present in simulations of these steps and was omitted for simplicity.

FEP details and results. NAMD's alchemical transformation module (in conjunction with the FEP method) and the collective variable module (for introduction of restraints) were used to implement the above transformations. The soft-core van der Waals radius-shifting coefficient (parameter alchVdwShiftCoeff) was set to $8 \AA^{2}$ in the first step of both transformations and to $20 \AA^{2}$ in the second step of both transformations (values were chosen to produce smooth transitions in short FEP test runs). All four steps were carried out using 20 FEP windows, with the coupling parameter varying uniformly from 0 to 1 . Each window involved $10 \mathrm{ps}$ of equilibration followed by $190 \mathrm{ps}$ of data collection. At the start of each simulation, the system (upon being minimized for 1,000 steps) was pre-equilibrated for 200 ps. Each step of both transformations was run 10 times in both forward and reverse directions, using different random seeds. Thus, a total of $336 \mathrm{~ns}$ of simulation was performed. The final results are summarized in Supplementary Fig. 8a, where values for reverse transformations have been negated to represent free energies in the decoupling direction. Error bars represent s.e.'s of the cumulative free-energy difference, computed over the 10 simulations run for each step/direction combination. Clearly, all steps exhibit excellent convergence and reversibility. The standard-state free energy of C60-COP binding was computed as:

$$
\Delta G_{1}^{\mathrm{I}}+\Delta G_{2}^{\mathrm{I}}-\left(\Delta G_{1}^{\mathrm{II}}+\Delta G_{2}^{\mathrm{II}}\right)+R T \ln \left[C^{\mathrm{o}}(2 \pi R T / k)^{3 / 2}\right]
$$

where $\Delta G_{i}^{\mathrm{K}}$ is the free-energy change of the $i$ th step of transformation $K$. The final estimate amounted to $-9.8 \pm 0.3 \mathrm{kcal} \mathrm{mol}^{-1}$, where the uncertainty was calculated by error propagation using s.e.'s emergent from combining all simulations of each step (both forward and reverse).

\section{Association of fullerene with individual aromatic groups. An analogous} approach was also used to calculate the affinity of $\mathrm{C}_{60}$ for a disembodied $\mathrm{Tyr}$ residue (acetylated and methyl-amidated on the $\mathrm{N}$ - and $\mathrm{C}$ termini, respectively) and a Tyr side-chain analogue ( $p$-methylphenol). The only difference was that in these cases an additional constant harmonic restraint, between the centre of mass of $\mathrm{C}_{60}$ and $\mathrm{C60D}$, was added throughout step 1 of transformation 1. This restraint, with a force constant of $1.0 \mathrm{kcal} \mathrm{mol}^{-1} \AA^{-2}$ and equilibrium distance of $0 \AA$, prevented $\mathrm{C}_{60}$ from dissociating from the bound molecule in the initial FEP window, which otherwise occasionally occurred in some trajectories and limited the amount of useful sampling. The effect of this restraint was removed from the final estimate by applying the standard importance sampling formula ${ }^{58}$ to adjust the expectation computed in $\mathrm{FEP}^{59}$. The final standard-state binding free-energy estimates were $-1.76 \pm 0.15$ for $\mathrm{C}_{60}$ and isolated $\mathrm{Tyr}$, and $-1.53 \pm 0.07$ for $\mathrm{C}_{60}$ and $p$-methylphenol (Supplementary Fig. $8 \mathrm{~b}-\mathrm{c}$ ). These correspond to dissociation constants in the $\mathrm{mM}$ range, meaning that the affinity is expected to be extremely weak.

Designability analysis. To estimate the natural abundance of structural motifs surrounding the $\mathrm{C}_{60}$-binding site, search engine MASTER ${ }^{33}$ (grigoryanlab.org/ master) was used to search a highly non-redundant subset of the PDB. Specifically, the weekly BLASTclust-based clustering ${ }^{60}$ of all PDB chains was downloaded on 22 October 2014, and the first chain from each cluster selected, filtering for X-ray structures resolved to $3 \AA$ or below. The asymmetric unit of each of the entries was then downloaded and the crystallographic lattice generated, keeping all images that were reasonably close to the initial unit (defined as having at least three atoms within $16 \AA$ of any atom in the initial unit). The resulting lattices were then combined into a MASTER database of 13,400 entries. All searches were performed using the full-backbone setting of MASTER, which provably finds the closest matches to the query in terms of the heavy-atom backbone r.m.s.d. (that is, N, CA, $\mathrm{C}$ and O). The full $\mathrm{C}_{60}$-binding motif was defined as residues 2-9 on one pair of chains and 19-24 on the opposing pair, with individual interfaces of this motif defined accordingly (Supplementary Fig. 7b). Sequence logos in Supplementary
Fig. 7b were generated by considering all matches within $0.3 \AA$ and discarding those with identical sequences (although the database is highly non-redundant, matches of identical sequence are still possible when multiple-matching instances are found within the same lattice).

Measurement of electrical conductance. Current versus voltage curves were obtained using the variable temperature microprobe system from MMR technologies coupled with HP 4145B semiconductor parameter analyser. The samples were deposited on a degenerately doped silicon substrate with 200The thermal oxide, which was photolithographically pre-patterned with $\mathrm{Au} / \mathrm{Cr}(45 \mathrm{~nm} /$ $5 \mathrm{~nm}$ ) electrodes. The channel length and width were 10 and $6,000 \mu \mathrm{m}$, respectively.

\section{References}

1. Talapin, D. V. \& Murray, C. B. PbSe nanocrystal solids for n- and p-channel thin film field-effect transistors. Science 310, 86-89 (2005).

2. Kumar, R. J., MacDonald, J. M., Singh, T. B., Waddington, L. J. \& Holmes, A. B. Hierarchical self-assembly of semiconductor functionalized peptide alphahelices and optoelectronic properties. J. Am. Chem. Soc. 133, 8564-8573 (2011).

3. Erb, R. M., Son, H. S., Samanta, B., Rotello, V. M. \& Yellen, B. B. Magnetic assembly of colloidal superstructures with multipole symmetry. Nature 457, 999-1002 (2009).

4. Koo, J. et al. Acentric 2-D ensembles of D-br-A electron-transfer chromophores via vectorial orientation within amphiphilic n-helix bundle peptides for photovoltaic device applications. Langmuir 28, 3227-3238 (2012).

5. Lu, Y. et al. Self-assembly of mesoscopically ordered chromatic polydiacetylene/ silica nanocomposites. Nature 410, 913-917 (2001).

6. Matthias, P. L., Penney, M. G. \& Helen, M. B. Designing materials to direct stem-cell fate. Nature 462, 433-441 (2009).

7. Zhang, J., Zheng, F. \& Grigoryan, G. Design and designability of protein-based assemblies. Curr. Opin. Struct. Biol. 27C, 79-86 (2014).

8. King, N. P. \& Lai, Y.-T. Practical approaches to designing novel protein assemblies. Curr. Opin. Struct. Biol. 23, 632-638 (2013).

9. King, N. P. et al. Computational design of self-assembling protein nanomaterials with atomic level accuracy. Science 336, 1171-1174 (2012)

10. King, N. P. et al. Accurate design of co-assembling multi-component protein nanomaterials. Nature 510, 103-108 (2014).

11. Grigoryan, G. et al. Computational design of virus-like protein assemblies on carbon nanotube surfaces. Science 332, 1071-1076 (2011)

12. Gonen, S., DiMaio, F., Gonen, T. \& Baker, D. Design of ordered twodimensional arrays mediated by noncovalent protein-protein interfaces. Science 348, 1365-1368 (2015).

13. Lanci, C. J. et al. Computational design of a protein crystal. Proc. Natl Acad. Sci. USA 109, 7304-7309 (2012)

14. Kaltofen, S. et al. Computational de novo design of a self-assembling peptide with predefined structure. J. Mol. Biol. 427, 550-562 (2015).

15. Egelman, E. H. et al. Structural plasticity of helical nanotubes based on coiled-coil assemblies. Structure 23, 280-289 (2015).

16. Xu, C. et al. Rational design of helical nanotubes from self-assembly of coiled-coil lock washers. J. Am. Chem. Soc. 135, 15565-15578 (2013).

17. Brodin, J. D. et al. Metal-directed, chemically tunable assembly of one-, two- and three-dimensional crystalline protein arrays. Nat. Chem. 4, 375-382 (2012).

18. Sinclair, J. C., Davies, K. M., Vénien-Bryan, C. \& Noble, M. E. Generation of protein lattices by fusing proteins with matching rotational symmetry. Nat. Nanotechnol. 6, 558-562 (2011).

19. Koga, N. et al. Principles for designing ideal protein structures. Nature 491, 222-227 (2012).

20. Friedman, S. H. et al. Inhibition of the HIV-1 protease by fullerene derivatives model building studies and experimental verification. J. Am. Chem. Soc. 115, 6506-6509 (1993).

21. Chen, B. X., Wilson, S. R., Das, M., Coughlin, D. J. \& Erlanger, B. F. Antigenicity of fullerenes: antibodies specific for fullerenes and their characteristics. Proc. Natl Acad. Sci. USA 95, 10809-10813 (1998).

22. Braden, B. C. et al. X-ray crystal structure of an anti-Buckminsterfullerene antibody fab fragment: biomolecular recognition of C(60). Proc. Natl Acad. Sci. USA 97, 12193-12197 (2000).

23. De Leo, F., Magistrato, A. \& Bonifazi, D. Interfacing proteins with graphitic nanomaterials: from spontaneous attraction to tailored assemblies. Chem. Soc. Rev. 44, 6916-6953 (2015).

24. Calvaresi, M. \& Zerbetto, F. Baiting proteins with C60. ACS Nano 4, 2283-2299 (2010).

25. Xie, L. et al. The molecular mechanism of fullerene-inhibited aggregation of Alzheimer's $\beta$-amyloid peptide fragment. Nanoscale 6, 9752-9762 (2014).

26. Georgakilas, V. et al. Supramolecular self-assembled fullerene nanostructures. Proc. Natl Acad. Sci. USA 99, 5075-5080 (2002). 
27. Meidine, M. F., Hitchcock, P. B., Kroto, H. W., Taylor, R. \& Walton, D. R. M. Single crystal X-ray structure of benzene-solvated C 60. J. Chem. Soc., Chemical Communications 1534-1537 (1992).

28. Yoko Nakajima, Y., Takeshi, Y., Kiyoshi, F., Syoko, S. \& Naoki, M. Solubilization of fullerenes into water with polyvinylpyrrolidone applicable to biological tests. J. Chem. Soc., Chem. Commun. 517-518 (1994).

29. Grigoryan, G. \& Keating, A. E. Structural specificity in coiled-coil interactions. Curr. Opin. Struct. Biol. 18, 477-483 (2008)

30. Grigoryan, G. \& Degrado, W. F. Probing designability via a generalized model of helical bundle geometry. J. Mol. Biol. 405, 1079-1100 (2011).

31. Gilson, M. K., Given, J. A., Bush, B. L. \& McCammon, J. A. The statisticalthermodynamic basis for computation of binding affinities: a critical review. Biophys. J. 72, 1047-1069 (1997).

32. Christ, C. D., Mark, A. E. \& van Gunsteren, W. F. Basic ingredients of free energy calculations: a review. J. Comput. Chem. 31, 1569-1582 (2010).

33. Zhou, J. \& Grigoryan, G. Rapid search for tertiary fragments reveals protein sequence-structure relationships. Protein Sci. 24, 508-524 (2015).

34. Franzen, S., Goldstein, R. F. \& Boxer, S. G. Distance dependence of electron-transfer reactions in organized systems: the role of superexchange and non-Condon effects in photosynthetic reaction centers. J. Phys. Chem. 97, 3040-3053 (1993).

35. Iversen, G., Kharkats, Y. I., Kuznetsov, A. M. \& Ulstrup, J. in Electron transfer from isolated molecules to biomolecules Advances in Chemical Physics (ed. Bixon, J. J.) 454-455 (John Wiley \& Sons, Inc., 1999).

36. Marshall, J. M. Carrier diffusion in amorphous-semiconductors. Rep. Prog. Phys. 46, 1235-1282 (1983).

37. Feng, M., Zhao, J. \& Petek, H. Atomlike, hollow-core-bound molecular orbitals of C-60. Science 320, 359-362 (2008).

38. DeGrado, W. F., Gratkowski, H. \& Lear, J. D. How do helix-helix interactions help determine the folds of membrane proteins? Perspectives from the study of homo-oligomeric helical bundles. Protein Sci. 12, 647-665 (2003).

39. Leslie, A. G. The integration of macromolecular diffraction data. Acta Crystallogr. D Biol. Crystallogr. 62, 48-57 (2006).

40. Powell, H. R. The Rossmann Fourier autoindexing algorithm in MOSFLM. Acta Crystallogr. D Biol. Crystallogr. 55, 1690-1695 (1999).

41. Evans, P. Scaling and assessment of data quality. Acta Crystallogr. D Biol. Crystallogr. 62, 72-82 (2006).

42. Otwinowski, Z. \& Minor, W. Processing of X-ray diffraction data collected in oscillation mode. Method Enzymol. 276, 307-326 (1997).

43. Matthews, B. W. Solvent content of protein crystals. J. Mol. Biol. 33, 491-497 (1968).

44. Mccoy, A. J. et al. Phaser crystallographic software. J. Appl. Crystallogr. 40, 658-674 (2007)

45. Adams, P. D. et al. PHENIX: a comprehensive Python-based system for macromolecular structure solution. Acta Crystallogr. D 66, 213-221 (2010).

46. Yeates, T. O. Simple statistics for intensity data from twinned specimens. Acta Crystallogr. A 44(Pt 2): 142-144 (1988).

47. Yeates, T. O. Detecting and overcoming crystal twinning. Methods Enzymol. 276, 344-358 (1997).

48. Fisher, R. G. \& Sweet, R. M. Treatment of diffraction data from crystals twinned by merohedry. Acta Crystallogr. A A36, 755-760 (1980).

49. Britton, D. Estimation of twinning parameter for twins with exactly superimposed reciprocal lattices. Acta Crystallogr. A A28, 296-297 (1972).

50. Emsley, P. \& Cowtan, K. Coot: model-building tools for molecular graphics. Acta Crystallogr. D 60, 2126-2132 (2004).

51. Phillips, J. C. et al. Scalable molecular dynamics with NAMD. J. Comput. Chem. 26, 1781-1802 (2005)

52. Brooks, B. R. et al. CHARMM: the biomolecular simulation program. J. Comput. Chem. 30, 1545-1614 (2009).

53. Yannoni, C. S., Bernier, P. P., Bethune, D. S., Meijer, G. \& Salem, J. R. NMR determination of the bond lengths in C60. J. Am. Chem. Soc. 113, 3190-3192 (1991).

54. Essmann, U. et al. A smooth particle mesh ewald method. J. Chem. Phys. 103, 8577-8593 (1995).
55. Shirts, M. R., Mobley, D. L., Chodera, J. D. \& Pande, V. S. Accurate and efficient corrections for missing dispersion interactions in molecular simulations. J. Phys. Chem. B 111, 13052-13063 (2007).

56. Shirts, M. R., Pitera, J. W., Swope, W. C. \& Pande, V. S. Extremely precise free energy calculations of amino acid side chain analogs: comparison of common molecular mechanics force fields for proteins. J. Chem. Phys. 119, 5740-5761 (2003).

57. Roux, B., Nina, M., Pomes, R. \& Smith, J. C. Thermodynamic stability of water molecules in the bacteriorhodopsin proton channel: a molecular dynamics free energy perturbation study. Biophys. J. 71, 670-681 (1996).

58. Surya, T. T. \& Robert, E. K. Importance sampling: a review. Wiley Interdiscip Rev.Comput. Stat. 2, 54-60 (2010).

59. William, L. J. \& Laura, L. T. Perspective on Free-energy perturbation calculations for chemical equilibria. J. Chem. Theory Comput. 4, 869-876 (2008).

60. Altschul, S. F., Gish, W., Miller, W., Myers, E. W. \& Lipman, D. J. Basic local alignment search tool. J. Mol. Biol. 215, 403-410 (1990).

\section{Acknowledgements}

We thank the staff at the MX beamline 7A-SBI (Pohang Light Source, Korea) and $\mathrm{X}$-ray diffraction facility at CCMB Hyderabad, India. This work was supported by Dartmouth College startup funds (G.G.), Alfred P Sloan Fellowship BR2013-038 (G.G.), Neukom Institute CompX Faculty grant (G.G.), National Institutes of Health (NIH) grant GM54616 (W.F.D.), National Science Foundation (NSF) grant CHE-1413295 (W.F.D.), NSF grant to the Laboratory for Research on the Structure of Matter (LRSM) of the University of Pennsylvania (DMR 1120901), National Research Foundation of Korea grants NRF-2014R1A1A2055647, NRF-2015M3C1A3002152, and IBS-R015-D1, the Agency for Defense Development through the Chemical and Biological Detection Research Center (CBDRC) (Y.H.K.), Ramalingaswami fellowship from Department of Biotechnology India (R.A.), National Institute of Science Education and Research startup funds (R.A.) and New Jersey Institute of Technology startup funds (D.-K.K).

\section{Author contributions}

K.-H.K, C.B.M., G.G., R.A., Y.H.K. and W.F.D. conceived and designed the study. K.-H.K. and N.H.K. synthesized the peptide. K.-H.K., N.H.K., J.P. and R.A. performed crystallization and data collection, and solved the crystal structures. G.G. designed and performed computational studies. S.-Q.Z. performed AUC experiment and analysed associated data. D.K., Y.-T.K. and C.B.M. characterized the electrical conductance. G.G, K.-H.K, R.A., Y.H.K. and W.F.D. integrated data and wrote the manuscript.

\section{Additional information}

Accession codes: The coordinates for the X-ray structures have been deposited to the Protein Data Bank (PDB) with accession codes 5ET3, 5HKN and $5 \mathrm{HKR}$

Supplementary Information accompanies this paper at http://www.nature.com/ naturecommunications

Competing financial interests: The authors declare no competing financial interests.

Reprints and permission information is available online at http://npg.nature.com/ reprintsandpermissions/

How to cite this article: Kim, K.-H. et al. Protein-directed self-assembly of a fullerene crystal. Nat. Commun. 7:11429 doi: 10.1038/ncomms11429 (2016).

This work is licensed under a Creative Commons Attribution 4.0 International License. The images or other third party material in this article are included in the article's Creative Commons license, unless indicated otherwise in the credit line; if the material is not included under the Creative Commons license, users will need to obtain permission from the license holder to reproduce the material To view a copy of this license, visit http://creativecommons.org/licenses/by/4.0/ 